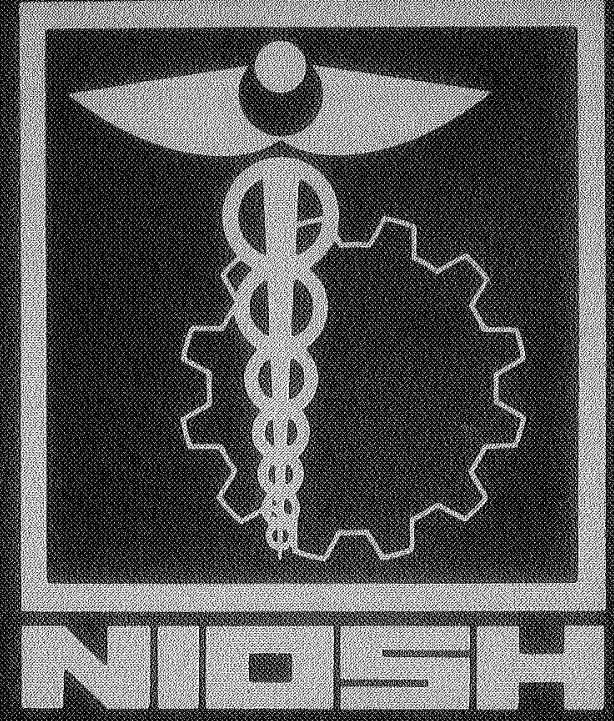

\title{
Proposed National Strategies for the Prevention of Leading Work - Related Diseases and Injuries
}

- Occupational Cardiovascular Diseases •

US. DEPAFTMENT OF FEALTH AND HUMAN SEFVICES

Public tealth Service Centers tor Disease Control Nationai institute tor Occupational Sarety and Healih 


\section{Proposed National Strategy for the Prevention of Occupational Cardiovascular Diseases}

U.S. DEPARTMENT OF HEALTH AND HUMAN SERVICES Public Health Service Centers for Disease Control National Institute for Occupational Safety and Health 
DHHS (NIOSH) Publication No. 89-132 


\section{Introduction}

This document, A Proposed National Strategy for the Prevention of Occupational Cardiovascular Diseases, summarizes what actions need to be taken to prevent occupational cardiovascular diseases. It was developed in 1985 at a conference sponsored by the National Institute for 0ccupational Safety and Health (NIOSH) and The Association of Schools of Public Health (ASPH), which brought together over 50 expert panelists and 450 other occupational safety and health professionals.

In addition to the strategy for occupational cardiovascular diseases, NIOSH and ASPH have published strategies for the other nine leading occupational diseases and injuries: occupational lung diseases, musculoskeletal injuries, occupational cancers, severe occupational traumatic injuries, disorders of reproduction, neurotoxic disorders, noise-induced hearing loss, dermatological conditions and psychological disorders.

The proposed strategies were originally published in a two volume set, Proposed National Strategies for the Prevention of Leading Work-Related Diseases and Injuries, Part 1 and Part 2. These proposed strategies are not to be considered as final statements of policy of NIOSH, The Association of Schools of Public Health, or of any agency or individual who was involved. Hopefully, they will be used in the quest to prevent disease and injury in the workplace.

To learn of the availability of the complete texts of Part 1 and Part 2, or to obtain additional copies of this or other Strategies, contact NIOSH Publications, 4676 Columbia Parkway, Cincinnati, Ohio 45226. Telephone (513) 533-8287. 


\section{A Proposed National Strafegy for the Prevention of Occupational Cardiovascular Diseases}

\section{Occupational Cardiovascular Diseases: Problems and Goals}

Cardiovascular diseases, including ischemic heart disease, hypertensive, cerebrovascular, and peripheral vascular diseases, constitute the leading cause of disability and death in the United States. In 1984, cardiovascular diseases accounted for almost a million deaths $(986,000)$, the majority $(555,000)$ from ischemic or coronary heart disease. The total economic costs (direct and indirect) from cardiovascular disease are substantial, amounting to approximately $\$ 102.4$ billion in 1983 . Since 1972 , coronary heart disease has declined by almost 34 percent. This decline is attributed to a decrease in several risk factors for cardiovascular disease in the population and to improved care for patients with cardiovascular diseases. These changes demonstrate the potential effectiveness of preventive programs directed at risk factors for cardiovascular disease.

Although cardiovascular diseases include a variety of diseases associated with different etiologic factors, several are related to common disease processes and common risk factors. Coronary heart disease, hypertension, and related disease entities are included in this prevention strategy because of their high incidence and prevalence in the United States. Other forms of cardiovascular disease, however, (e.g., arrhythmias, cardiomyopathies) are more directly related to specific occupational exposures or conditions. Although these latter conditions are less common, they receive specific emphasis in this strategy because of their more direct relationship to occupational factors and their resultant vulnerability to intervention in the workplace.

\section{Risks and the Workplace}

Much is already known about the personal risk factors associated with an increased incidence of coronary heart disease. Major alterable contributors to risk of this disease include cigarette smoking, dietary intake (particularly in relationship to levels of blood lipids), and hypertension. Age, gender, and family history are also important, but not alterable. Other factors include excessive alcohol intake, obesity, diabetes, inadequate physical activity, and behavioral pattern. The available evi- 
dence indicates that preventive programs directed at alterable risk factors are already effective in reducing the occurrence of cardiovascular disease. Programs directed at cessation of cigarette smoking, management of hypertension, and change in diet appear to be particularly important.

The workplace can have a deleterious effect on cardiovascular health. A review of existing information on the relationship between workplace factors and cardiovascular disease indicates that millions of workers are currently exposed to work-related factors associated with an increased risk of cardiovascular disease. These factors include exposure to chemical and physical agents in the workplace as well as workrelated psychosocial factors. Thus, while the workplace contains factors hazardous to cardiovascular health, it also contains opportunities to prevent and control risk factors for cardiovascular disease. Studies have shown that the workplace is an excellent site for reaching workers with prevention and intervention messages and programs, especially those aimed at reducing personal risk factors for cardiovascular disease.

In this strategy for preventing cardiovascular disease in the workplace, we propose where possible and more efficacious to combine the two approaches: preventing work-related risk factors and enhancing the prevention of personal risk factors. Where it is possible to combine these approaches into an integrated strategy, they offer prospects for substantially reducing the present occurrence of cardiovascular disease.

\section{A. Cardiotoxic Exposures in the Workplace}

1. Available Information

Occupational exposures to several chemical and physical agents are known to increase the risk of cardiovascular disease or its manifestations. These agents include carbon disulfide, carbon monoxide, halogenated hydrocarbons, nitroglycerin and related compounds, heat stress, and noise. Occupational exposures to these agents are associated with different manifestations of cardiovascular disease, including immediate deaths from exposures to carbon monoxide or heat stress, acute arrhythmias from exposure to halogenated hydrocarbons, and the development of chronic cardiovascular disease from exposure to carbon disulfide. This variation in the manifestation of specific cardiovascular disease is important to recognize both in controlling these problems and in conducting further research.

Other occupational exposures that may increase the risk of cardiovascular disease include cobalt, lead, antimony, and cadmium. A review of Criteria Documents and Occupational Health Guidelines from the National Institute for Occupational Safety and Health (NIOSH) identified 85 substances with possible cardiotoxic effects. A search of the NIOSH Registry of Toxic Effects of Chemical Substances (RTECS) showed 1,466 chemicals with possible cardiovascular effects. Epidemiologic studies have also identified increased risk of cardiovascular disease for a number of occupational groups, such as clerical workers, fire fighters, and bus and truck drivers. This information provides the basis for a more systematic evaluation of both the substances and the occupational groups to better delineate the cardiovascular risks from such exposures. 
Because the cardiovascular disease process is complex, appropriate research methodologies must be carefully developed to study the relationship between occupational exposures and cardiovascular disease. In general, results of research on the relationships of work to cardiovascular health have been less definitive than those for research in many other areas of occupational health, partly because conducting studies in this area is difficult: 1) coronary heart disease has a long latency period during which occupational exposures may vary greatly for an individual, 2) in most epidemiologic studies that have examined personal risk factors, historical information on occupational exposures was not collected systematically, 3) most standard occupational mortality studies have failed to obtain information on personal risk factors (e.g., smoking) or to adjust for selection factors among workers (i.e., "healthy worker effect"), and 4) the complexities of the disease process also make ordinary toxicologic studies quite difficult to design and conduct.

\section{What Can Be Done Now}

\section{a. Health Protection: Reducing the Environmental Risks}

Several steps can be taken to reduce the risks of cardiovascular disease among workers exposed to substances known to increase this risk. Such workers can be identified by examining existing environmental surveillance data (National Occupational Exposure Survey, OSHA inspection records, etc.). Specific programs can be designed to reduce exposures to cardiovascular toxins in these settings. Potential prevention programs should address the need for better communication to the exposed workers about the known hazards (including the development of better methods of conducting this communication); better compliance with current exposure criteria; development and installation of better control technology to reduce specific cardiotoxic exposures; development of better environmental control programs, including improved environmental and medical monitoring; development of better protective equipment; and adoption of new or improved standards for specific exposures (e.g., heat stress, methylene chloride) now known to be hazardous. These programs need to provide for ongoing surveillance of both exposures to these substances and the related incidence of cardiovascular disease; they need to identify new industrial exposures to cardiotoxic agents and new control methods.

\section{b. Health Promotion: Enhancing Healthy Behaviors}

As a general rule, programs for health promotion and disease prevention-including the reduction of risk factors for cardiovascular diseaseshould be initiated at the worksite whenever possible or practical. When environmental factors increase the risk of cardiovascular disease, the need and value of these worksite programs becomes even more pronounced. In an ideal situation, both environmental and personal risks would be addressed through appropriate strategies at the workplace. Programs that address environmental risks should include efforts to reduce personal, modifiable risk factors for cardiovascular diseasesmoking, elevated blood cholesterol, elevated blood pressure, sedentary lifestyle, and others. This combined approach offers a favorable interactive effect for reducing the occurrence of cardiovascular disease among workers exposed to agents such as carbon disulfide. Encouraging workers to quit smoking is particularly important because smoking contributes not 
only to several cardiovascular diseases but to other diseases as well. Preventive efforts aimed at reducing personal risk factors should be brought to the work setting to ensure that involved workers have full and effective access to them.

However, a combined strategy is not always possible and could even result in a negative effect on both. Some situations may dictate that one set of risks be addressed before the other without hampering the effectiveness of either. For example, cardiotoxic hazards may be so severe in a plant that they must be addressed before efforts are implemented for personal risk factors. Alternately, cardiotoxins may be minimal or even nonexistent in a workplace, and thus it would not be feasible or even reasonable to implement anything but a program for control of personal risk factors.

\section{What Knowledge Do We Need}

As mentioned previously, better medical, epidemiologic, and toxicologic studies are needed of a number of substances and physical agents to determine their effects on the cardiovascular system in various occupational groups. Given the scant attention paid to occupational factors in past studies of cardiovascular disease, these factors should play a large role in new investigations. Development at NIOSH of an epidemiologic group for cardiovascular disease would provide a useful focus and serve to coordinate this work at NIOSH. This group could also coordinate a program with other agencies and outside groups. Several specific studies are readily suggested for this NIOSH group, including defining the conditions under which arrhythmias occur following exposure to fluorinated hydrocarbons and the interaction of this effect with such factors as caffeine intake; determining whether chronic exposure to carbon monoxide causes cardiovascular disease; evaluating possible cardiomyopathy following exposure to cobalt, taking diet and alcohol intake into account; assessing the effects of antimony on the cardiovascular system; better defining the relationship between hypertension and exposure to noise; and examining several large studies on the risk of cardiovascular disease to determine possible effects of occupational risk. Occupational health researchers must be urged to use available, well-established methods to assess personal risk factors.

If the assessment of occupational exposures in large studies of personal risk factors for cardiovascular disease can be improved (i.e., a standard occupational questionnaire), those studies will be very helpful in assessing occupational risk factors. Coordination with the National Heart, Lung, and Blood Institute (particularly their new series of prospective studies) and with other groups devoted to the epidemiology of cardiovascular disease will also be important in this effort.

A parallel effort should be made to improve methods for toxicologic assessment of possible cardiotoxic substances. Short-term tests are needed to screen substances for their effects on the cardiovascular system, and techniques should be developed for delineating mechanisms of toxicity.

Further research is also required in the related areas of developing new monitoring techniques to assess exposures to cardiotoxic agents (e.g., continuous monitoring of fluorocarbon exposures) and assessing the value of the 
training, education, and information dissemination that is directed toward lowering the risk of cardiovascular disease in the workplace.

\section{B. Psychosocial Factors}

\section{Available Information}

Several studies have shown an association of work-related psychosocial factors with an increased risk of cardiovascular disease. In reviewing these studies, it is important to note that the associations often apply only to certain manifestations of cardiovascular disease. For example, work requiring extended vigilance with severe penalties for mistakes (e.g., air traffic controlling) is associated with an increased risk of hypertension but not of other manifestations of cardiovascular disease. Work overload (e.g., being employed in two jobs) is associated with an increased risk of developing angina pectoris and myocardial infarctions but not of sudden death or hypertension. Other psychosocial factors that appear to affect some forms of cardiovascular disease include personality type, socioeconomic status, anxiety, sleep disturbances, life changes, and the administrative organization of the workplace.

\section{What Can Be Done Now}

Many studies of associations between work-related psychosocial factors and cardiovascular disease are difficult to interpret, and not all attempts to replicate the findings have yielded consistent results. This may be explained in part by the use of slightly different methods in studies that measured similar psychosocial factors and by the frequent lack of controls for other risk factors of cardiovascular disease. Thus, although these studies demonstrate the importance of work-related psychosocial factors in the risk of cardiovascular disease, the specific underlying factors that cause this increased risk are difficult to define. Therefore, our strategy calls for further research in this area rather than the application of specific preventive actions at this time.

\section{What Knowledge Do We Need}

More information is badly needed on the association between work-related psychosocial factors and the development of cardiovascular disease. This research could focus on several factors, such as machine pacing of work, work overload (e.g., holding two jobs), work organization, job-decision latitude, and various aspects of stress at work. The studies should be done carefully and should control for the effects of other cardiovascular risk factors.

Better methods should be developed and applied to assess workplace psychosocial factors. New technologies that may increase job-related stress (particularly computer-mediated work) should be carefully assessed to limit their adverse effects on workers. Research is also needed to determine the effectiveness of programs that are implemented to correct work-related psychosocial factors (e.g., job redesign, relaxation training).

\section{Health Promotion: Role of the Workplace}

1. Available Information

The prevention strategies outlined here are directed at specific groups that 
may be at increased risk from occupational exposures or psychosocial factors encountered in the workplace. Intervention programs directed at personal risk factors are an important aspect of the strategy for dealing with these occupational cardiovascular problems. However, for the many occupational and industrial groups with increased risk primarily from personal factors, the prevention of cardiovascular disease related to these personal risk factors is also an important public health goal.

The workplace is a highly attractive site for delivering health promotion and employee-assistance programs because of certain characteristics of many occupational settings and the high prevalence of many personal risk factors for cardiovascular disease. These programs are rapidly becoming widespread in different industries because they offer the hope of effectively reducing both personal and medical care costs. Programs directed at hypertension control, smoking cessation, and problems of alcohol and other substance abuse appear to provide good models with demonstrated effectiveness. Many industries have also introduced programs focused on dietary changes and physical fitness.

Several factors that are important for the success of health promotion programs at the worksite include management and union cooperation, employee involvement in the program, adequate allocation of resources, and control of the program at the local plant level. Attention to ethical issues is also important in the management of these programs. Programs should be voluntary and should maintain the confidentiality of any medical information collected. All data collected on environmental and personal risk factors should be disclosed to the participants. When programs to control personal risk factors are being conducted in the workplace, environmental hazards there should also be appropriately addressed through the usual mechanisms. In some circumstances, control of environmental and personal risk factors may be addressed through an integrated program.

\section{What Can Be Done Now}

Efforts' should be made to increase the availability of health promotion programs in the workplace. This requires the cooperation of industry, labor unions, community and voluntary groups, and the government. These efforts should include the identification of groups at high risk of cardiovascular and other diseases based on exposure in the workplace and personal risk factors. Effective programs for different industrial and occupational groups should be developed to extend these programs to as many people as possible. The programs should focus on smoking cessation, control of hypertension, dietary changes, physical fitness, and employee assistance for alcohol abuse and relate? problems. Structured followup and evaluations should be included in individual programs. Although most programs will be conducted in the private sector, governmental agencies can play an important role in stimulating the development of effective programs and in evaluating them.

\section{What Knowledge Do We Need}

Much information is needed to assess the effectiveness of health promotion programs in the workplace, particularly for different occupational groups and different approaches to these groups. The enormous increase in the preva- 
lence of these programs does not confirm their effectiveness; popularity is not the same as efficacy.

The use of occupationally oriented health risk appraisals specifically needs evaluation. Successful worksite-intervention programs that have been limited to particular industries should be evaluated for possible broader application, such as identifying differing approaches based on the size of the industry and the nature of the worksite.

\section{Summary}

Because of the extent of morbidity and mortality from cardiovascular disease in this country, implementation of this prevention strategy is very important. This effort will require the participation of many groups including industry, labor unions, governmental agencies, and voluntary groups (particularly the American Heart Association). Although our knowledge of the relationship between the workplace and cardiovascular disease is incomplete, several steps can be taken now to reduce this risk; moreover, considerable research is needed to better assess the risk. Implementing an integrated strategy that involves both health protection-to control workplace exposures, and health promotion-to control personal factors that increase the risk of cardiovascular disease, could substantially improve the aggregate health of our workforce. 


\section{Contributors}

NIOSH WORKING GROUP MEMBERS

James M. Melius, M.D., Dr. P.H., Chair Rebecca Stanevich, R.N.

Charles Althafer, M.P.H.

Mark A. Torrason, Ph.D.

William H. Perry

Kenneth Weber, Ph.D.

Cynthia Robinson

Mary L. Woebkenberg

Lawrence M. Schleifer, Ed.D.

SYMPOSIUM PANELISTS

Philip J. Landrigan, M.D., Chair

National Institute for Occupational Safety and Health

Duane L. Block, M.D.

Ford Motor Company

Sidney Green, Ph.D.

Food and Drug Administration

William R. Harlan, M.D.

University of Michigan Medical Center

William L. Hopkins, Ph.D.

University of Kansas

C. David Jenkins, Ph.D.

University of Texas Medical Branch
Franklin Mirer, Ph.D.

United Auto Workers

David P. Rall, M.D., Ph.D.

National Institutes of Health

Jonathan S. Raymond, Ph.D.

University of Hawaii

Kenneth D. Rosenman, M.D.

New Jersey State Department of Health

Sheldon W. Samuels

AFL-CiO 